\title{
In NPH, setting valve opening pressure close to lumbar puncture opening pressure decreases overdrainage
}

\author{
Tito Vivas-Buitrago ${ }^{1}$, Ricardo Domingo ${ }^{1}$, Shashwat Tripathi ${ }^{1}$, Juan Pablo Herrera ${ }^{1}$, Johan Heemskerk ${ }^{1}$, \\ Sanjeet Grewal ${ }^{1}$, Nicholas L. Zalewski ${ }^{2}$, Alfredo Quinones-Hinojosa ${ }^{1}$, Ronald Reimer ${ }^{1}$, \\ Robert E. Wharen ${ }^{1}$, Neill R. Graff-Radford ${ }^{3}$ \\ ${ }^{1}$ Department of Neurosurgery, Mayo Clinic, Jacksonville, Florida, USA \\ ${ }^{2}$ Department of Neurology, Mayo Clinic, Rochester, Florida, USA \\ ${ }^{3}$ Department of Neurology, Mayo Clinic, Jacksonville, Florida, USA
}

\begin{abstract}
Background. The management of normal pressure hydrocephalus (NPH) can be difficult, partly because there are frequent treatment complications such as overdrainage which, when serious, may require surgical intervention. We previously reported a correlation between the difference of lumbar puncture opening pressure minus the valve opening pressure setting (LPOP VOP) (which we refer to as the delta) and increased rates of overdrainage. This led to a modification in our practice, whereby we now set the VOP equal to, or close to, the LPOP, resulting in lower deltas.

Objective. In this new study, our aim was to compare the rate of overdrainage in our patients with higher and lower deltas and assess the significance of setting the VOP equal, or close, to the patient's LPOP.

Methods. 1. We reproduced the association between delta and overdrainage. 2 . We compared the incidence of overdrainage in those whose VOP was set close to LPOP (low delta) versus those with VOP setting distant from the LPOP (higher delta). 3. We compared symptom improvement in those with a low versus higher delta.

Results. We confirmed the relation between high delta and an increased rate of overdrainage, lower rates of overdrainage in those whose VOP was set close to the LPOP (Delta Adjusted Practice), and better improvement of symptoms when the VOP was set closer to the LPOP.
\end{abstract}

Conclusion. We propose that the initial VOP should be set as close as possible to the patient's LPOP to decrease overdrainage without compromising symptom improvement.

Key words: NPH, shunting, lumbar puncture opening pressure, initial shunt valve setting, delta, overdrainage

(Neurol Neurochir Pol 2020; 54 (6): 531-537)

\section{Introduction}

Treatment-related complications of normal pressure hydrocephalus (NPH) adds to the already complex management of this condition. A common complication is overdrainage (subdural haematoma, subdural hygroma and postural headache) [1-16].

Reports have indicated that low valve opening pressure (VOP) settings increase the chance of overdrainage [17, 18]. Boon et al. and Delwel et al. conducted randomised trials comparing low vs. medium/high valve settings on shunted patients diagnosed with NPH, and reported comparable symptom improvement, but decreased rates of overdrainage in $\mathrm{pa}$ tients with an initial medium/high VOP. Nontheless, patients with higher initial VOPs required subsequent follow-up visits to slowly lower the valve opening pressure, in the pursuit of symptom improvement with less risk of overdrainage $[17,18]$.

In 2013, we reported that overdrainage is related to the difference between the lumbar puncture opening pressure (LPOP) and the initial valve opening pressure (VOP) [19]. 
The larger this difference (which we called the delta), the more frequent the problem.

Two important factors contributed to this:

1. Before there were adjustable shunts, neurosurgeons had to choose a shunt with a preset valve opening pressure. The most commonly used valve was the medium pressure valve which has an opening pressure of $120 \mathrm{~mm}$ of $\mathrm{H}_{2} \mathrm{O}$. Even when adjustable valves became available, the initial valve opening pressure was set at $120 \mathrm{~mm}$ of $\mathrm{H}_{2} \mathrm{O}[17,18]$.

2. Also in the 2013 study, we demonstrated a correlation between Body Mass Index (BMI) and LPOP, with patients with increased BMI having high LPOP [19]. Thus, the delta between the LPOP and the VOP was frequently high. This in turn correlated with overdrainage complications [19]. Subsequent to this report, two of our neurosurgeons modified their practice to set the VOP close to the LPOP. In this current study, our aims were:

1. To reproduce the association between delta and overdrainage analysis

2. To compare the incidence of overdrainage in those whose VOP was set close to LPOP (low delta) versus those with VOP setting distant from the LPOP (higher delta)

3. To compare symptom improvement in those with a low versus higher delta.

\section{Methods}

\section{Patient selection}

This study was approved by our institutional review board. No patient consent was required, given the retrospective nature of the study and the anonymisation of the data. 222 electronic records from patients diagnosed with iNPH treated at our institution betwen January 2005 and October 2018 were reviewed. The following variables were extracted: age, sex, symptoms at presentation, time since symptom onset, BMI, imaging findings, LPOP, VOP, date of first valve adjustment, radiological overdrainage (subdural haematoma or hygroma as seen on postoperative CT scans), clinical overdrainage (postural headache), serious overdrainage (subdural collection requiring surgical evacuation), other complications, and any improvement noted (general, gait, memory, or urine control) after shunt placement.

\section{Pre-operative assessment}

iNPH diagnosis and indication for shunt placement was made based on clinical and radiological findings. A high-volume lumbar puncture for gait assessment as a predictor of clinical improvement after shunt placement was performed, thus avoiding more invasive testing such as external lumbar drainage or intraventricular pressure monitoring. Thirty millilitres of CSF were drained with the patient in the lateral decubitus position with legs, back, and limbs flexed. The back was positioned perpendicular to the edge of the bed, with the hips and legs parallel to each other. Gait improvement was determined either by gait evaluation $30 \mathrm{~min}$ after the procedure or by family reports of improvement over the subsequent 24 hours. Surgical procedure of choice consisted on a ventriculo-peritoneal shunt placement of a programmable valve without anti-siphon device.

\section{Delta effects on outcome}

LPOP and VOP values were extracted from patient medical records. The delta was defined as the difference between the lumbar puncture opening pressure and the initial valve opening pressure (LPOP minus VOP). Delta was then evaluated for associations with patient outcomes (gait, memory, and urinary) and overdrainage rates.

\section{Delta adjusted vs non-delta adjusted treatment paradigm}

The standard treatment for iNPH is shunt placement with an initial VOP of $120 \mathrm{mmH}_{2} \mathrm{O}[17,18]$. However, previous work by our group showed that a lower delta (LPOP - VOP) was associated with a lower incidence of overdrainage, which led us to the hypothesis that setting the initial VOP close to the LPOP would result in less overdrainage while maintaining shunt symptom improvement.

In this current study, delta was divided into $10 \mathrm{mmH}_{2} \mathrm{O}$ intervals because this is the smallest interval between valve opening pressure settings. Patients were additionally categorised as being either NDA (treated under the non-delta adjusted protocol with a delta greater than $10 \mathrm{mmH}_{2} \mathrm{O}$ ) or DA (where VOP was set close to LPOP, with a delta less than $+/-10 \mathrm{mmH}_{2} \mathrm{O}$ ). We then compared the clincal and radiographic outcomes from the immediate postoperative course after shunt placement to the time of first valve adjustment between NDA and DA groups.

\section{Statistical analysis}

T-test and linear regressions were used to analyse factors associated with the delta. Logistic regression was used to test the effect of delta on the presence of shunt placement outcomes. Significance was considered at $\alpha \leq 0.05$ (two-sided). $R$ (version 3.6.0) was utilised to analyse patient data. Next, statistically significant variables on univariate analysis were included in a multivariate model to analyse their impact on overdrainage. In order to account for potential confounding factors, differences in radiographic overdrainage rates based on surgical experience ( $>20$ years of experience $v s$ $<20$ years), and time between LPOP measurement and shunt placement, were calculated.

\section{Results}

Of the 222 patients we reviewed, 13 were removed from further analysis because they were taking anticoagulation/antiplatelet medication, or presented to an emergency department following a fall during which they hit their head, resulting in subdural haematomas. The median age of the remaining 209 patients was 77 years (IQR 73-80); 128 were male and 
the median BMI was 29 (IQR 26-33) (Tab. 1). The median LPOP was $150 \mathrm{mmH}_{2} \mathrm{O}$ (IQR 120-170), the median delta (LPOP minus VOP) was $10 \mathrm{mmH}_{2} \mathrm{O}$ (IQR -10-30) (Tab. 1). Radiographic overdrainage rates were not statistically different based on surgical experience $(<20$ years experience $=20 \% v s>20$ years experience $=16 \% ; \mathrm{P}=0.45)$, or based on time between LPOP and shunt placement (overdrainage $=1.43$ months vs non-overdrainage $=2.05$ months; $\mathrm{P}=0.14$ ).

Table 1. Demographics, symptom outcome and overdrainage

\begin{tabular}{|c|c|}
\hline Demographics & $\mathbf{N}(\%)$ \\
\hline Number of patients & $209(100 \%)$ \\
\hline Age & $77(73-80)^{*}$ \\
\hline Sex (female) & $81(40 \%)$ \\
\hline BMI & $29(26-33)^{*}$ \\
\hline Lumbar puncture opening pressure & $150(120-127)^{*}$ \\
\hline Number of patients with VOP at 120 & $68(33 \%)$ \\
\hline \multicolumn{2}{|l|}{ Symptom outcome } \\
\hline Gait difficulty prior to shunt & $209(100 \%)$ \\
\hline Gait improvement after shunt ${ }^{\circledR}$ & $200 / 209(96 \%)$ \\
\hline Memory loss prior to shunt & $133(64 \%)$ \\
\hline Memory improvement after shunt ${ }^{\bigotimes}$ & $100 / 133(76 \%)$ \\
\hline Urinary difficulty prior to shunt & $148(71 \%)$ \\
\hline Urinary improvement after shunt $^{\bigotimes}$ & $126 / 148(85 \%)$ \\
\hline \multicolumn{2}{|l|}{ Overdrainage complications } \\
\hline Radiographic overdrainage & $37(18 \%)$ \\
\hline Subdural hygroma & $32(15 \%)$ \\
\hline Subdural haematoma & $9(4 \%)$ \\
\hline Subdural hygroma + haematoma & $4(2 \%)$ \\
\hline Serious overdrainage ${ }^{* *}$ & $4(2 \%)$ \\
\hline $\begin{array}{l}\text { Median (IQR) } \\
\text { *Of those presenting with radiographic overdrainage } \\
\text { Associations with improvement after shunt placement } \\
\text { ifficulty prior to shunt placement }\end{array}$ & ts with \\
\hline
\end{tabular}

\section{Patients outcomes after shunting}

Two-hundred and nine patients $(100 \%)$ presented with gait difficulties prior to shunt, $133(64 \%)$ with memory loss, and $148(69 \%)$ with urinary difficulties. After shunting, by patient and doctor consenus in the chart 200 (96\%) showed gait improvement, $100(76 \%)$ had memory improvements, and $126(85 \%)$ had urinary improvement (Tab. 1). Additionally, $75(38 \%)$ had postural headaches, and 37 (18\%) had radiographic overdrainage prior to first valve adjustment. Of these 37 with radiographic overdrainage, only four (11\%) had serious overdrainage that required surgery for subdural evacuation (Tab. 1).

\section{Delta effects on outcome}

Patient demographics, symptom outcomes, and overdrainage are set out in Table 1. Age and sex were not significantly associated with delta. BMI however was significantly associated with delta, with higher BMI leading to higher delta (P-value $<0.01$ ) (Tab. 2). Univariate logistic analysis revealed that higher delta increased the risk for radiographic overdrainage (Odds Ratio $(\mathrm{OR})=1.2$ (CI 1.1-1.2), P-value $=0.002)($ Tab. 2, Fig. 1) and risk for serious overdrainage $(\mathrm{OR}=1.3$ (CI 1.1-1.5), P-value $=0.03)($ Tab. 2, Fig. 1). Additionally, higher delta reduced the chance of memory improvements $(\mathrm{OR}=0.88(\mathrm{CI}$ 0.84-0.94), P-value $=0.03)($ Tab. 2, Fig. 2) and urinary improvements $(\mathrm{OR}=0.98(\mathrm{CI} 0.97-0.99), \mathrm{P}$-value $=0.02)(\mathrm{Tab} .2$, Fig. 3). No significant relation was found between delta and gait improvement $(\mathrm{OR}=1.0(\mathrm{CI} 0.9-1.1), \mathrm{P}$-value $=0.90)($ Tab. 2, Fig. 4). In our cohort, each $10 \mathrm{mmH}_{2} \mathrm{O}$ increase in delta led to a $12 \%$ and a $2 \%$ lower chance of memory and urinary improvement, respectively, and a $20 \%$ greater chance of radiological overdrainage and a $30 \%$ greater chance of serious overdrainage. Moreover, multivariate analysis found that higher delta pressure increased the odds for radiographic overdrainage, even after accounting for all significant variables on univariate analysis. $(\mathrm{OR}=1.05$ (CI 1.01-1.09, $\mathrm{P}<0.01)$ (Tab. 3).

Table 2. Association of demographics and shunt placement outcomes with delta (VOP-LPOP) in 10 mmH20 increments) using univariate analysis

\begin{tabular}{|c|c|c|}
\hline Demographics* & Regression coefficient $(95 \% \mathrm{Cl})$ & P-value \\
\hline Age & $-0.2(-0.5-0.1)$ & 0.62 \\
\hline Sex (baseline $=$ female) & $1.0(-3.6-5.6)$ & 0.84 \\
\hline BMI & $1.1(0.5-1.7)$ & $<0.01$ \\
\hline Symptom improvement** & Odds ratio $(95 \% \mathrm{CI})$ & P-value \\
\hline Gait improvement after shunt $[n=200 / 209(96 \%)]^{\dagger}$ & $1.0(0.9-1.1)$ & 0.90 \\
\hline Memory improvement after shunt $[n=100 / 133(76 \%)]^{\dagger}$ & $0.88(0.84-0.94)$ & 0.03 \\
\hline Urinary improvement after shunt $[n=126 / 148(85 \%)]^{\dagger}$ & $0.98(0.97-0.99)$ & 0.02 \\
\hline Overdrainage** & Odds ratio $(95 \% \mathrm{CI})$ & P-value \\
\hline Radiographic overdrainage $[n=37(18 \%)]$ & $1.2(1.1-1.2)$ & 0.002 \\
\hline Serious overdrainage $[n=4 / 37(11 \%)]^{* * *}$ & $1.3(1.1-1.5)$ & 0.03 \\
\hline
\end{tabular}

*Based on linear regression with delta pressure as outcome and respective predictor

**Based on logistic regression with delta pressure as predictor

***Of those presenting with radiographic overdrainage who required surgery for evacuation

${ }^{\dagger}$ Associations with improvement after shunt placement were only explored in patients with difficulty prior to shunt placement 


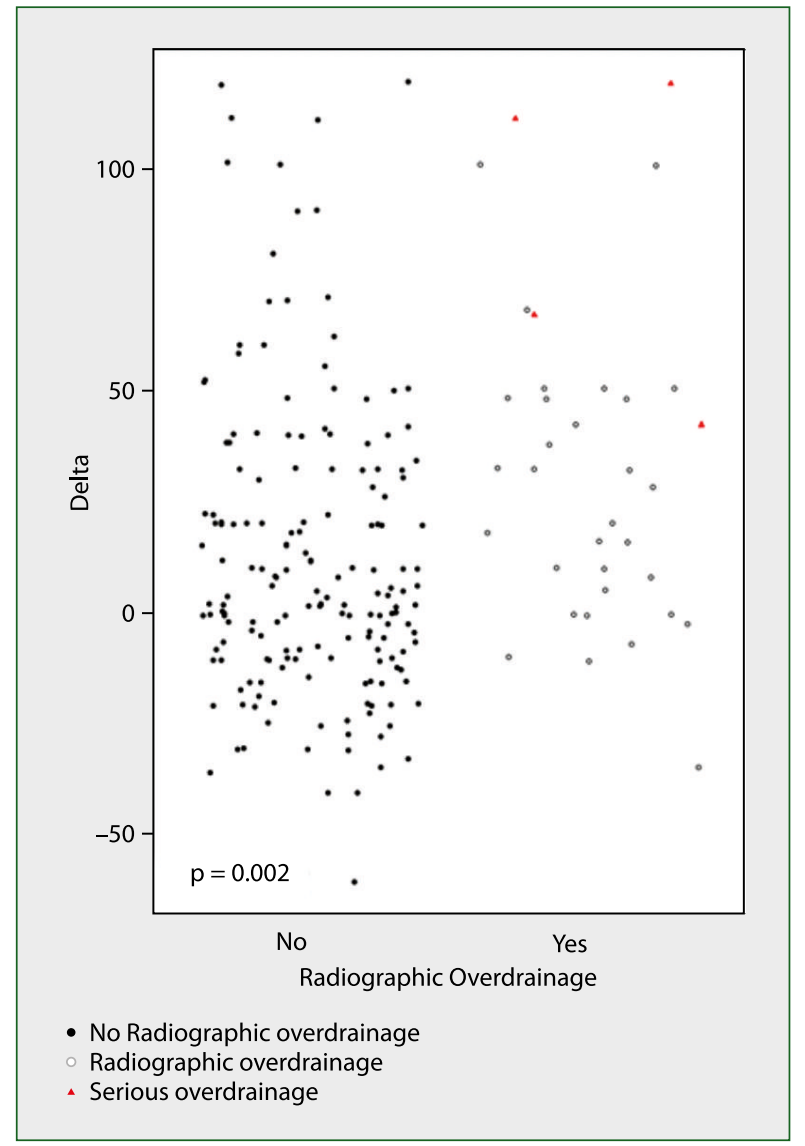

Figure 1. Boxplot showing delta pressure according to presence/absence of radiographic overdrainage. Red triangle = serious overdrainage

\section{Delta adjusted vs non-delta adjusted treatment paradigm}

The DA $(n=76)$ and the NDA ( $\mathrm{n}=133)$ groups did not differ in any demographic parameter (age, sex, BMI, or LPOP). DA patient surgery dates ranged from December 2005 to May 2018; those of NDA patients ranged from January 2005 to October 2018. We found that DA patients had significantly lower radiological overdrainage (DA $8 \%$ vs NDA $23 \%, \mathrm{P}=<0.01$ ) (Tab. 4, Fig. 5). Serious overdrainage rates were lower for DA patients, although this was not statistically significant (DA $0 \%$ vs NDA $13 \%$, $\mathrm{P}=0.08)$ (Tab. 4, Fig. 5). Additionally, there was no difference in the improvement percentages between NDA and DA practices (Tab. 4).

\section{Discussion}

Patients in this study presented with an overall positive symptom outcome after shunt implantation regardless of the LPOP, VOP, or delta values (Tab. 1, 2). Although symptom recovery was evident when shunting these patients, the important rates of radiological overdrainage and serious overdrainage cannot be ignored.

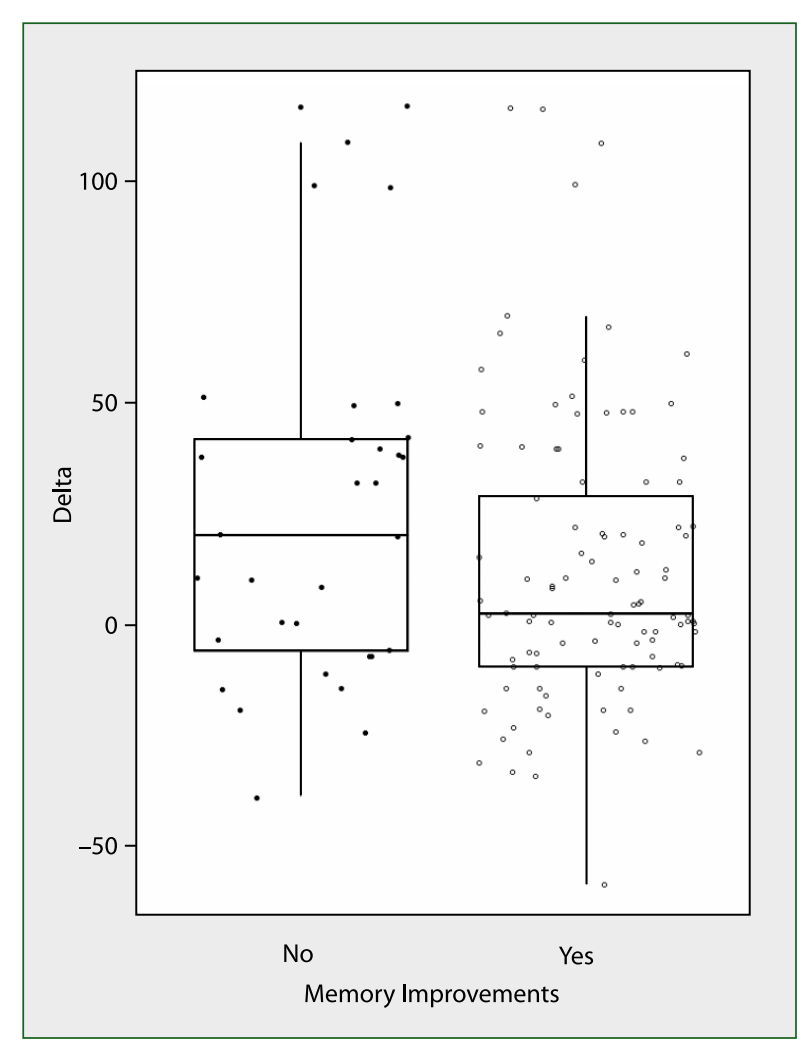

Figure 2. Boxplot showing delta pressure according to presence / absence of memory improvements

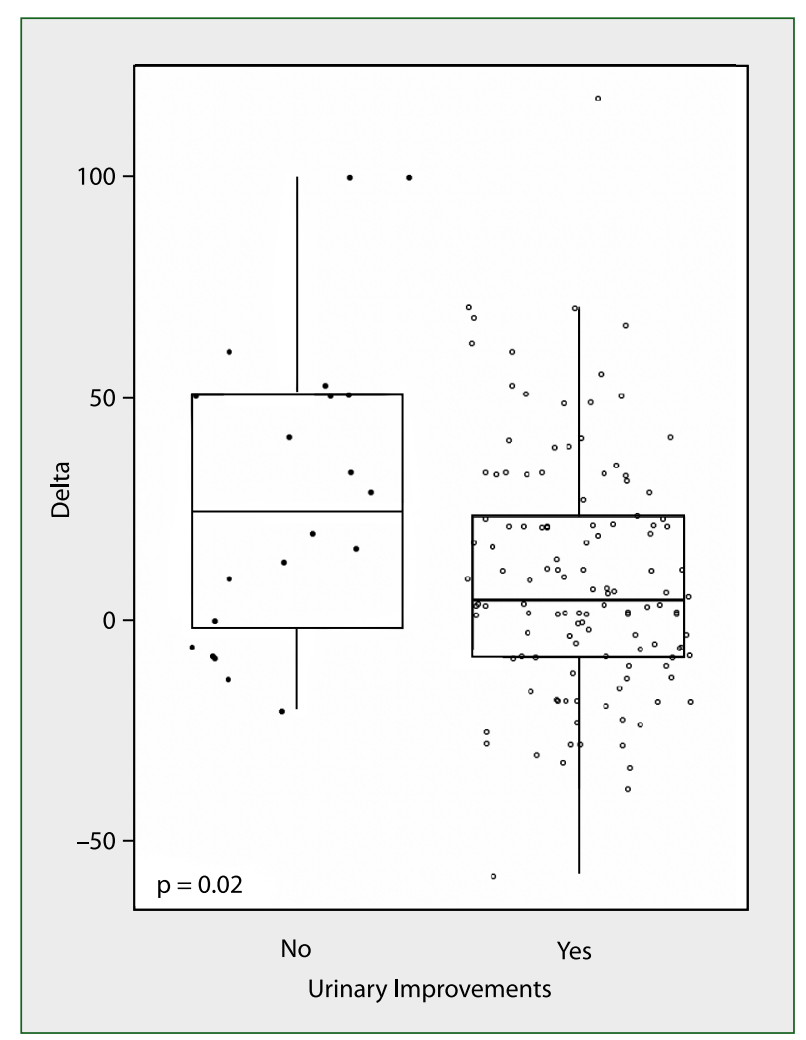

Figure 3. Boxplot showing delta pressure according to presence / absence of urinary improvements 


\section{Delta effects on outcome}

Our analysis of delta's effect on outcomes showed that higher delta considerably increased the risk of radiographic

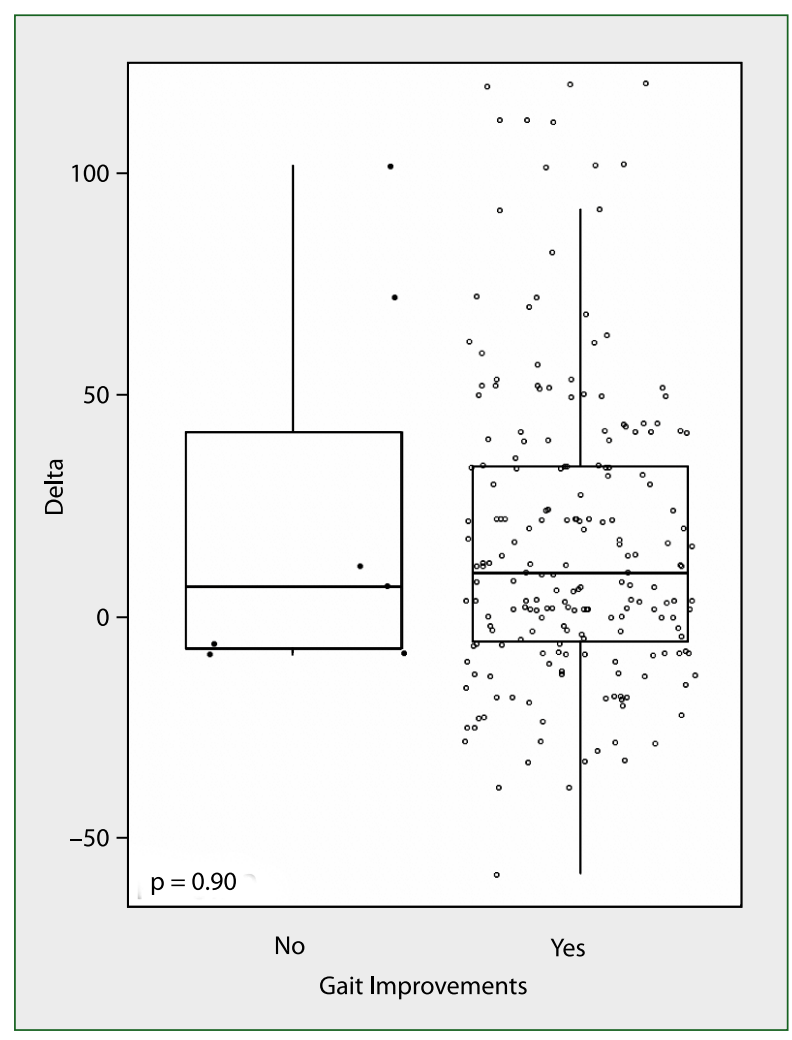

Figure 4. Boxplot showing delta pressure according to presence / absence of gait improvements

Table 3: Multivariate analysis of risk factors for radiographic overdrainage

\begin{tabular}{lcc}
\multicolumn{1}{c}{ Variables } & Odds ratio $(95 \% \mathrm{Cl})$ & P-value \\
\hline $\mathrm{BMI}$ & $1.1(1.04-1.15)$ & 0.02 \\
$\begin{array}{l}\text { Delta pressure }(10 \\
\text { mmH2O increments) }\end{array}$ & $1.05(1.01-1.09)$ & $<0.01$ \\
\hline
\end{tabular}

and serious overdrainage (a 20\% greater chance of radiological overdrainage and a $30 \%$ greater chance of serious overdrainage). Also, higher deltas had a lower chance of memory and urinary improvement (for every $10 \mathrm{mmH}_{2} \mathrm{O}$ increase in delta, there was a $12 \%$ and a $2 \%$ lower chance of memory and urinary improvement, respectively). Although statistically significant associations on clinical outcomes were observed, the clinical

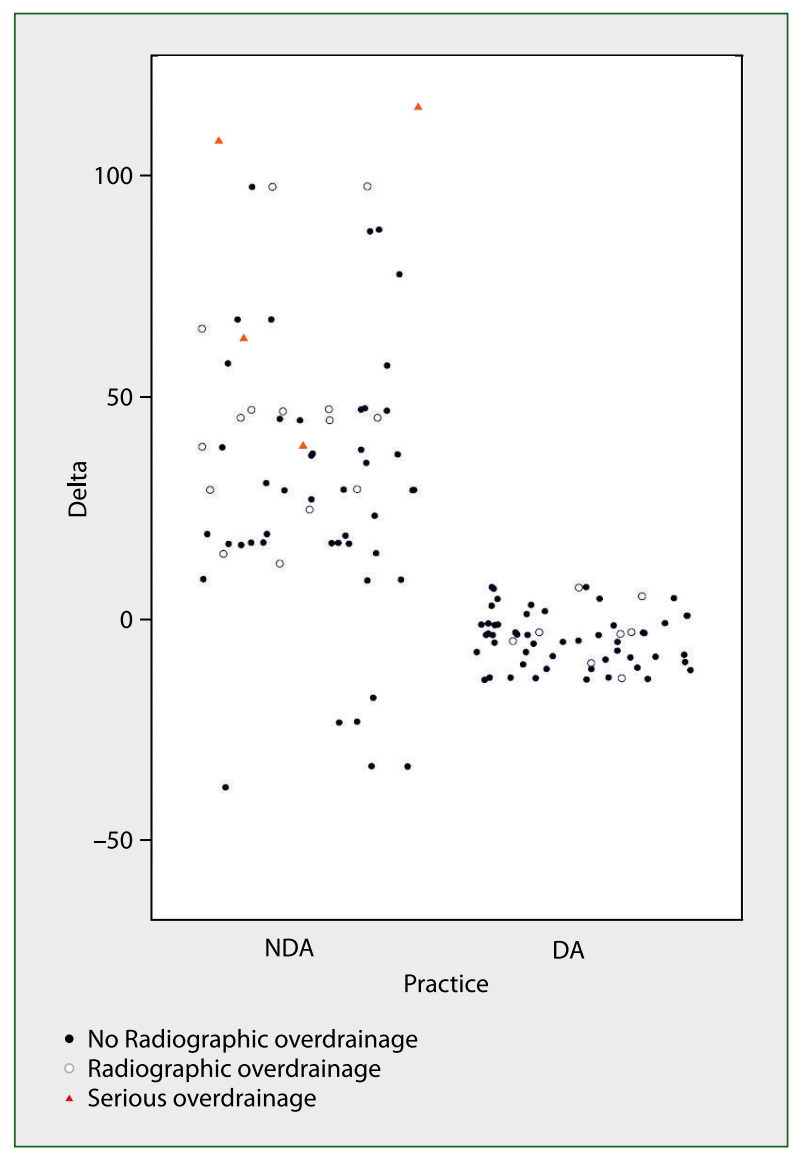

Figure 5. Plot showing delta pressure according to presence / absence of radiographic improvements. Red triangle $=$ serious overdrainage. DA - Delta adjusted, NDA - Non-delta adjusted

Table 4. Association of overdrainage with non-delta adjusted (NDA) vs delta adjusted (DA) practice

\begin{tabular}{|c|c|c|c|}
\hline Overdrainage type & NDA ( $N=133)$ & $\mathrm{DA}(\mathbf{N}=76)$ & P-Value \\
\hline Age & $75.0(73-79)$ & $75.6(71-83)$ & 0.54 \\
\hline Sex (female) & $49(37 \%)$ & $30(39 \%)$ & 0.82 \\
\hline Gait improvement after shunt ${ }^{\rrbracket}$ & $128(96 \%)$ & $72(92 \%)$ & 0.60 \\
\hline Memory improvement after shunt ${ }^{\rrbracket}$ & $62 / 88(70 \%)$ & $39 / 51(76 \%)$ & 0.57 \\
\hline Urinary improvement after shunt ${ }^{\llbracket}$ & $77 / 96(80 \%)$ & $46 / 52(88 \%)$ & 0.30 \\
\hline Radiographic overdrainage & $31(23 \%)$ & $6(8 \%)$ & $<0.01$ \\
\hline Serious overdrainage $\mathrm{e}^{* * *}$ & $4(13 \%)$ & $0(0 \%)$ & 0.08 \\
\hline
\end{tabular}

Delta adjusted (DA) practice: ventricle opening pressure set within $10(+/-5) \mathrm{mmH}_{2} \mathrm{O}$ to lumbar puncture opening pressure

Non-delta adjusted (NDA) practice: ventricle opening pressure set at $120 \mathrm{mmH}_{2} \mathrm{O}$ regardless of LP pressure

*Based on linear regression with delta pressure as outcome and respective predictor

**Based on logistic regression with delta pressure as predictor

***Of those presenting with radiographic overdrainage

${ }^{\dagger}$ Associations with improvement after shunt placement were only explored in patients with difficulty prior to shunt placement 
implications must be cautiously interpreted since outcomes were assessed based on subjective data, and were therefore prone to report bias. Futhermore, we found no difference in gait improvement associated with the delta value. This is probably related to the limited size of the unimproved group $(n=9,5 \%)$, comprising five patients in the NDA group and four patients in the DA group.

\section{Delta adjusted vs non-delta adjusted treatment paradigm}

As a result of our previous publication [19], two neurosurgeons in our institution decided to modify their practice by placing the initial shunt valve settings equal to the LPOP. And, in light of the abovementioned findings, our group decided to further evaluate the effects of delta in patient outcomes by comparing the results from NDA treatment practices to those of DA treatment practices. After classifying patient care as being either DA or NDA, no significant differences in terms of demographics, age, BMI, or LPOP were identified between the two groups.

We found no significant differences in patient symptom recovery between NDA vs DA practice (Tab. 3). However, we identified that patients who underwent treatment defined as DA practice had significantly lower rates of radiographic overdrainage compared to NDA practice (Tab. 3, Fig. 5). Additionally, although not significantly, four cases of serious overdrainage were identified in the NDA practice group, vs. zero cases in the DA practice group (Tab. 3, Fig. 5). We found a significant decrease in overdrainage when initially setting the valve opening pressure equal to or within $<10 \mathrm{mmH}_{2} \mathrm{O}$ of the LPOP.

Patients with iNPH have been treated since the 1960s with similar methods that have relied on CSF deviation techniques with significant rates of symptom improvement $[4,8,9,12$, 20]. Although positive outcomes after shunting are very well documented, the high surgical complication rate has introduced a note of caution before recommending shunt surgery [21-23]. The more we can decrease the complication rate, the less doctors will hesitate before recommending surgery.

The limitations of our study are that this is not a randomised blinded prospective study but a retrospective analysis of patients at one site with potential variability due to the involvement of different surgeons performing procedures, as well as the possibility of selection bias and inaccurate or incomplete data at time of collection.

Furthermore, we did not have a quantitative measure of improvement but rather relied on documentation of the consensus of patient and physician. Therefore, the applicability of our findings to other cohorts may be limited.

Nevertheless, it confirms our original observation that increased LPOP minus VOP is related to overdrainage, causing a change in our practice to set the VOP as close as possible to the LPOP to provide a patient-specific protocol, thereby decreasing the risk of overdrainage without compromising symptom improvement.
Finally, no correction for multiple comparisons was conducted, although it has been noted that this should not impact upon the results we present here [24].

\section{Conclusion}

This study reports a significant correlation of LPOP minus VOP on overdrainage complications and symptom outcomes. We propose that the initial VOP should be set as close as possible to the patient's LPOP to decrease overdrainage without compromising symptom improvement.

Conflict of interest: None.

Funding: None.

\section{References}

1. Adams RD, Fisher CM, Hakim S, et al. Symptomatic occult hydrocephalus with "normal" cerebrospinal-fluid pressure.a treatable syndrome. N Engl J Med. 1965; 273: 117-126, doi: 10.1056/ NEJM196507152730301, indexed in Pubmed: 14303656.

2. Duinkerke A, Williams MA, Rigamonti $D$, et al. Cognitive recovery in idiopathic normal pressure hydrocephalus after shunt. Cogn Behav Neurol. 2004; 17(3): 179-184, doi: 10.1097/01. wnn.0000124916.16017.6a, indexed in Pubmed: 15536306.

3. Graff-Radford NR, Jones DT. Normal Pressure Hydrocephalus. Continuum (Minneap Minn). 2019; 25(1): 165-186, doi: 10.1212/ CON.0000000000000689, indexed in Pubmed: 30707192.

4. Hakim S, Adams RD. The special clinical problem of symptomatic hydrocephalus with normal cerebrospinal fluid pressure. Observations on cerebrospinal fluid hydrodynamics. J Neurol Sci. 1965; 2(4): 307-327, doi: 10.1016/0022-510x(65)90016-x, indexed in Pubmed: 588917.

5. Hung AL, Vivas-Buitrago T, Adam A, et al. Ventriculoatrial versus ventriculoperitoneal shunt complications in idiopathic normal pressure hydrocephalus. Clin Neurol Neurosurg. 2017; 157: 1-6, doi: 10.1016/j. clineuro.2017.03.014, indexed in Pubmed: 28347957.

6. Jusué-Torres I, Jeon LH, Sankey EW, et al. A Novel Experimental Animal Model of Adult Chronic Hydrocephalus. Neurosurgery. 2016; 79(5): 746-756, doi: 10.1227/NEU.0000000000001405, indexed in Pubmed: 27759679.

7. Kiefer M, Eymann R, Steudel W. Outcome redictors for normal-ressure hydrocehalus. Brain Edema XIII: Sringer; 2006. p. : 364-7.

8. Klinge $P$, Marmarou $A$, Bergsneider $M$, et al. Outcome of shunting in idiopathic normal-pressure hydrocephalus and the value of outcome assessment in shunted patients. Neurosurgery. 2005; 57(3 Suppl): S40-52; discussion ii, doi: 10.1227/01.neu.0000168187.01077.2f, indexed in Pubmed: 16160428.

9. McGirt MJ, Woodworth G, Coon AL, et al. Diagnosis, treatment, and analysis of long-term outcomes in idiopathic normal-pressure hydrocephalus. Neurosurgery. 2005; 57(4): 699-705; discussion 699, doi: 10.1093/neurosurgery/57.4.699, indexed in Pubmed: 16239882.

10. McGovern RA, Kelly KM, Chan AK, et al. Should ventriculoatrial shunting be the procedure of choice for normal-pressure hydrocephalus? J Neurosurg. 2014; 120(6): 1458-1464, doi: 10.3171/2014.1.JNS131808, indexed in Pubmed: 24605842.

11. Murakami $Y$, Matsumoto $Y$, Hoshi $K$, et al. Rapid increase of "brain-type' transferrin in cerebrospinal fluid after shunt surgery for idiopathic normal pressure hydrocephalus: a prognosis marker for cognitive re- 
covery. J Biochem. 2018; 164(3): 205-213, doi: 10.1093/jb/mvy043, indexed in Pubmed: 29701803.

12. Pujari S, Kharkar S, Metellus $P$, et al. Normal pressure hydrocephalus: long-term outcome after shunt surgery. J Neurol Neurosurg Psychiatry. 2008; 79(11): 1282-1286, doi: 10.1136/jnnp.2007.123620, indexed in Pubmed: 18356257.

13. Vivas-Buitrago T, Lokossou A, Jusué-Torres I, et al. Aqueductal Cerebrospinal Fluid Stroke Volume Flow in a Rodent Model of Chronic Communicating Hydrocephalus: Establishing a Homogeneous Study Population for Cerebrospinal Fluid Dynamics Exploration. World Neurosurg. 2019; 128: e1118-e1125, doi: 10.1016/j.wneu.2019.05.093, indexed in Pubmed: 31121363.

14. Vivas-Buitrago T, Pinilla-Monsalve G, Jusué-Torres I, et al. Ventricular Volume Dynamics During the Development of Adult Chronic Communicating Hydrocephalus in a Rodent Model. World Neurosurg. 2018; 120: e1120-e1127, doi: 10.1016/j.wneu.2018.08.241, indexed in Pubmed: 30217783.

15. Wu EM, El Ahmadieh TY, Kafka B, et al. Ventriculoperitoneal Shunt Outcomes of Normal Pressure Hydrocephalus: A Case Series of 116 Patients. Cureus. 2019; 11(3): e4170, doi: 10.7759/cureus.4170, indexed in Pubmed: 31093469.

16. Esmonde T, Cooke S. Shunting for normal pressure hydrocephaIus (NPH). Cochrane Database Syst Rev. 2002(3): CD003157, doi: 10.1002/14651858.CD003157, indexed in Pubmed: 12137677.

17. Boon AJ, Tans JT, Delwel EJ, et al. Dutch Normal-Pressure Hydrocephalus Study: randomized comparison of low- and medium-pressure shunts. J Neurosurg. 1998; 88(3): 490-495, doi: 10.3171/ jns.1998.88.3.0490, indexed in Pubmed: 9488303.
18. Delwel EJ, de Jo, Dammers R, et al. van den Brink W, Dirven CM. $A$ randomised trial of high and low pressure level settings on an adjustable ventriculoperitoneal shunt valve for idiopathic normal pressure hydrocephalus: results of the Dutch evaluation programme Strata shunt (DEPSS) trial. Journal of neurology, neurosurgery, and psychiatry. 2013; 84(7): 813-7.

19. Khan QU, Wharen RE, Grewal SS, et al. Overdrainage shunt complications in idiopathic normal-pressure hydrocephalus and lumbar puncture opening pressure. J Neurosurg. 2013; 119(6): 1498-1502, doi: 10.3171/2013.7.JNS13484, indexed in Pubmed: 23930853.

20. Kang YS, Park EK, Kim JS, et al. Efficacy of endoscopic third ventriculostomy in old aged patients with normal pressure hydrocephalus. Neurol Neurochir Pol. 2018; 52(1): 29-34, doi: 10.1016/j. pjnns.2017.10.004, indexed in Pubmed: 29103634.

21. Vanneste J, Augustijn P, Dirven C, et al. Shunting normal-pressure hydrocephalus: do the benefits outweigh the risks? A multicenter study and literature review. Neurology. 1992; 42(1): 54-59, doi: 10.1212/ wnl.42.1.54, indexed in Pubmed: 1734324.

22. Saper CB. The Emperor has no clothes. Ann Neurol. 2016; 79(2): 165-166, doi: 10.1002/ana.24603, indexed in Pubmed: 26788859.

23. Sharkey P, Pinilla-Monsalve G, Rigamonti A, et al. Health Care Expenditures of Medicare Beneficiaries with Normal Pressure Hydrocephalus. World Neurosurg. 2019; 127: e548e555, doi: 10.1016/j.wneu.2019.03.201, indexed in Pubmed: 30928595.

24. Rothman KJ. No adjustments are needed for multiple comparisons. Epidemiology. 1990; 1(1): 43-46, indexed in Pubmed: 2081237. 